Received: 18.05 .2018

Revised: 15.06 .2018

Accepted: 29.06 .2018

DOI: $10.17804 / 2410-9908.2018 .3 .043-057$

\title{
BOUNDARY LAYER INFLUENCE ON THE DISTRIBUTION OF HYDROGEN CONCENTRATIONS DURING HYDROGEN-INDUCED CRACKING TEST OF STEELS
}

\author{
E. L. Alekseeva ${ }^{1, \text { a) }}$, A. K. Belyaev ${ }^{1,2, \text { b) }}$, A. S. Zegzhda ${ }^{1, \text { c) }}$, A. M. Polyanskiy ${ }^{3, \text { d), }}$
} V. A. Polyanskiy ${ }^{1,2, e^{*}}$, K. P. Frolova ${ }^{1,2, \text { f) }}$, Yu. A. Yakovlev ${ }^{1,2, g)}$

${ }^{1}$ Peter the Great St. Petersburg Polytechnic University, 29 Politekhnicheskaya, St. Petersburg, 195294, Russian Federation

${ }^{2}$ Institute of Problems of Mechanical Engineering RAS,

61 V.O., Bolshoy Ave., St., St. Petersburg, 199178, Russian Federation

${ }^{3}$ RDC Electron \& Beam Technology, Ltd.,

6 Bronevaya St., St. Petersburg, 198188, Russian Federation

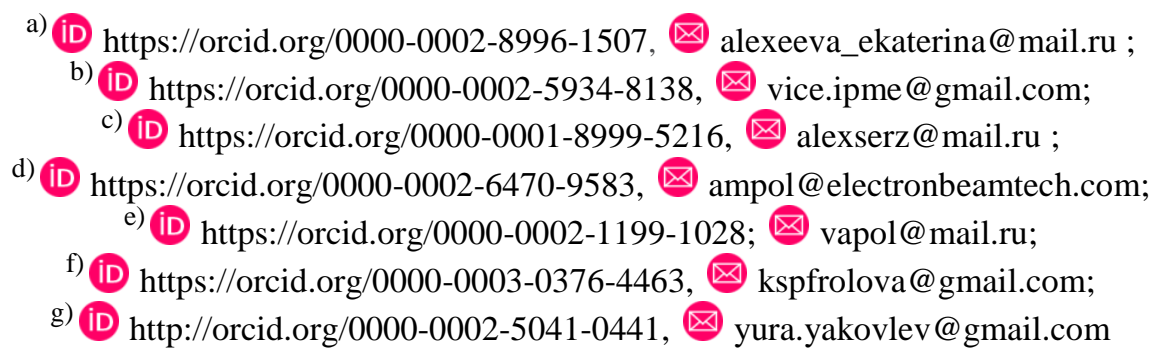

*Corresponding author. E-mail: vapol@mail.ru

Address for correspondence: V.O., Bolshoj pr., 61, St. Petersburg 199178, Russian Federation

Tel.: +7-921-7480637; fax: +7-812-3214771

Hydrogen saturation is a recognized and standardized method for testing the propensity to hydrogen-induced cracking and corrosion resistance of metals. Hydrogen saturation in a neutral salt solution simulating seawater and groundwater during the bubbling of the solution with hydrogen sulfide, as well as in the electrolyte by the cathodic current of hydrogen ions, are the main industrial test procedures. These tests are necessary for steels used, for example, in gas and oil pipelines. In this paper, we explain results of investigation of hydrogen concentration distribution after such testing.

The method of direct measurements has revealed a significant heterogeneity of hydrogen concentrations. This observation leads to concern about the suitability of industrial testing methods for real operation conditions for construction components in an aggressive environment.

Keywords: hydrogen saturation, hydrogen induced cracking, steels testing.

\section{Acknowledgment}

The study was supported by RFBR, projects 17-08-00783, 18-08-00201 and 18-31-00329.

\section{References}

1. Hadfield R. The occlusion of gases by metals. A general discussion. Introductory address. Transactions of the Faraday Society, 1919, vol. 14, pp. 173-191.

2. Johnson W.H. On some remarkable changes produced in iron and steel by the action of hydrogen and acids. In: Proceedings of the Royal Society of London, 1874, vol. 23, no. 156-163, pp. 168-179. 
3. Andrew J.H. The embrittling of iron by caustic soda. Transactions of the Faraday Society, 1914, vol. 9, pp. 316-329.

4. Keiichi O. On the importancy of hydrogen-brittleness as a defect in steel qualities. Tetsu-to-Hagane, 1938, vol. 24, no. 11, pp. 1005-1013. DOI: 10.2355/tetsutohagane1915.24.11_1005.

5. BS ISO 16573:2015. Steel. Measurement method for the evaluation of hydrogen embrittlement resistance of high strength steels.

6. ISO 11114-4. Transportable gas cylinders compatibility of cylinder and valve materials with gas contents. Part 4: Test methods for selecting metallic materials resistant to hydrogen embrittlement, 2005.

7. ISO 17081:2014. Method of measurement of hydrogen permeation and determination of hydrogen uptake and transport in metals by an electrochemical technique, 2014.

8. ANSI/NACE TM0284. Evaluation of Pipeline and Pressure Vessel Steel for Resistance to hydrogen-Induced Crack, 2016, no. 21215.

9. Akiyama E., Li S. Electrochemical hydrogen permeation tests under galvanostatic hydrogen charging conditions conventionally used for hydrogen embrittlement study. Corrosion Reviews, 2016, vol. 34, iss. 1-2, pp. 103-112. DOI: 10.1515/corrrev-2015-0049.

10. Omura T., Nakamura J., Hirata H., Jotoku K., Ueyama M., Osuki T., Terunuma M. Effect of surface hydrogen concentration on hydrogen embrittlement properties of stainless steels and Ni based alloys. ISIJ International, 2016, vol. 56, no. 3, pp. 405-412. DOI: 10.2355/isijinternational.ISIJINT-2015-268.

11. Hadam U., Zakroczymski T. Absorption of hydrogen in tensile strained iron and high-carbon steel studied by electrochemical permeation and desorption techniques. International Journal of Hydrogen Energy, 2009, vol. 34, no. 5, pp. 2449-2459. DOI: 10.1016/j.ijhydene.2008.12.088.

12. Martinsson A., Sandstrum R. Hydrogen depth prole in phosphorus-doped, oxygen - free copper after cathodic charging. Journal of Materials Science, 2012, vol. 47, no. 19, pp. 6768-6776. DOI: $10.1007 / \mathrm{s} 10853-012-6592-\mathrm{y}$.

13. Yagodzinskyy Y., Todoshchenko O., Papula S., Hдnninen H. Hydrogen solubility and diffusion in austenitic stainless steels studied with thermal desorption spectroscopy. Steel Research International, 2011, vol. 82, no. 1, pp. 20-25. DOI: 10.1002/srin.201000227.

14. Wu R., Ahlstrцm J., Magnusson H., Frisk K., Martinsson A. Charging, degassing and distribution of hydrogen in cast iron. Swerea KIMAB, 2015, vol. 1, no. 14, pp. 1-41.

15. Konopel'ko L.A., Polyanskii A.M., Polyanskii V. A., Yakovlev Yu.A. New Metrological Support for Measurements of the Concentration of Hydrogen in Solid Samples. Measurement Techniques, 2018, vol. 60, no. 12, pp. 1222-1227. DOI: 10.1007/s11018-018-1343-3.

16. Konar J., Banerjee N.G. Vacuum heating hydrogen determination in aluminium and aluminium alloys. NML Technical Journal, 1974, vol. 16, no. 1-2, pp 18-19.

17. Belyaev A.K., Polyanskiy A.M., Polyanskiy V.A., Sommitsch Ch., Yakovlev Yu.A. Multichannel diffusion vs TDS model on example of energy spectra of bound hydrogen in 34CrNiMo6 steel after a typical heat treatment. International Journal of Hydrogen Energy, 2016, vol. 41, no. 20, pp. 8627-8634. DOI: 10.1016/j.ijhydene.2016.03.198.

18. Polyanskiy A.M., Polyanskiy V.A., Yakovlev Yu.A. Experimental determination of parameters of multichannel hydrogen diffusion in solid probe. International Journal of Hydrogen Energy, 2014, vol. 39, no. 30, pp. 17381-17390. DOI: 10.1016/j.ijhydene.2014.07.080.

19. Andronov D.Yu., Arseniev D.G., Polyanskiy A.M., Polyanskiy V.A., Yakovlev Yu.A. Application of multichannel diusion model to analysis of hydrogen measurements in solid. International Journal of Hydrogen Energy, 2017, vol. 42, no. 1, pp. 699-710. DOI: 10.1016/j.ijhydene.2016.10.126.

20. Ryu J.H., Chun Y.S., Lee C.S., Bhadeshia H.K.D.H., Suh D.W. Effect of deformation on hydrogen trapping and effusion in trip-assisted steel. Acta Materialia, 2012, vol. 60, no. 10, pp. 4085-4092. DOI: 10.1016/j.actamat.2012.04.010. 
21. Choo W.Y., Lee J.Y. Thermal analysis of trapped hydrogen in pure iron. Metallurgical Transactions A, 1982, vol. 13, no. 1, pp. 135-140. DOI: 10.1007/BF02642424.

22. Ramunni V.P., De Paiva Coelho T., De Miranda P.E.V. Interaction of hydrogen with the microstructure of low-carbon steel. Materials Science and Engineering: A, 2006, vol. 435, pp. 504-514. DOI: 10.1016/j.msea.2006.07.089.

23. Kim C.D., Loginow A.W. Techniques for investigating hydrogen-induced cracking of steels with high yield strength. Corrosion, 1968, vol. 24, no. 10, pp. 313-318. DOI: 10.5006/0010-931224.10.313.

24. Newman J.F., Shreir L.L. The effect of temperature upon the solubility and diffusion coefficient of cathodic H2 in steel. Corrosion Science, 1971, vol. 11, no. 1, pp. 25-33. DOI: $10.1016 / \mathrm{S} 0010-938 X(71) 80071-9$.

25. Farrell K. Cathodic hydrogen absorption and severe embrittlement in a high strength steel. Corrosion, 1970, vol. 26, no. 3, pp 105-110. DOI: 10.5006/0010-9312-26.3.105.

26. Chan S.L.I. Hydrogen trapping ability of steels with different microstructures. Journal of the Chinese Institute of Engineers, 1999, vol. 22, no. 1, pp. 43-53. DOI: 10.1080/02533839.1999.9670440.

27. Chan S.L.I., Charles J.A. Effect of carbon content on hydrogen occlusivity and embrittlement of ferrite-pearlite steels. Materials Science and Technology, 1986, vol. 2, no. 9, pp. 956-962. DOI: 10.1179/mst.1986.2.9.956.

28. Choo W.Y., Lee J.Y., Cho C.G., Hwang S.H. Hydrogen solubility in pure iron and effects of alloying elements on the solubility in the temperature range 20 to 500 C. Journal of Materials Science, 1981, vol. 16, no. 5, pp. 1285-1292. DOI: 10.1007/BF01033843.

29. Jenkins N., Hart P., Parker D.H. An evaluation of rapid methods for diffusible weld hydrogen. Welding Journal-Including Welding Research Supplement, 1997, vol. 76, no. 1, pp. 1-6.

30. Lunarska E., Flis J. Effect of hydrogen charging-discharging on the stress-strain relationship for nickel. Scripta Metallurgica, 1984, vol. 18, no. 9, pp. 889-892. DOI: 10.1016/00369748(84)90255-2.

31. Lunarska E. Changes in the strain hardening rate of iron and steel during absorption and desorption of hydrogen under cathodic charging and discharging conditions. Scripta Metallurgica, 1981, vol. 15, no. 10, pp. 1077-1082. DOI: 10.1016/0036-9748(81)90161-7.

32. Pearson W.B. Miscellaneous aspects of lattice-spacing variation. Chapter V. In: W.B. Pearson. A Handbook of Lattice Spacing and Structures of Metals and Alloys: International Series of Monographs on Metal Physics and Physical Metallurgy. Vol. 4. Oxford, Pergamon, 1958, pp. $68-75$.

33. Hu Z., Fukuyama S., Yokogawa K., Okamoto S. Hydrogen embrittlement of a single crystal of iron on a nanometre scale at a crack tip by molecular dynamics. Modelling and Simulation in Materials Science and Engineering, 1999, vol. 7, no. 4, p. 541.

34. Pressouyre G.M. Trap theory of hydrogen embrittlement. Acta Metallurgica, 1980, vol. 28, no. 7, pp. 895-911. DOI: 10.1016/0001-6160(80)90106-6.

35. Song J., Curtin W.A. A nanoscale mechanism of hydrogen embrittlement in metals. Acta Materialia, 2011, vol. 59, no. 4, pp. 1557-1569. DOI: 10.1016/j.actamat.2010.11.019.

36. Chen Y., Zheng S., Zhou J., Wang P., Chen L., Qi Y. Influence of H2S interaction with prestrain on the mechanical properties of high-strength X80 steel. International Journal of Hydrogen Energy, 2016, vol. 41, no. 24, pp. 10412-10420. DOI: 10.1016/j.ijhydene.2016.01.144.

37. Sanchez J., Lee S.F., Martin-Rengel M.A., Fullea J., Andrade C., Ruiz-Hervias J. Measurement of hydrogen and embrittlement of high strength steels. Engineering Failure Analysis, 2016, vol. 59, pp. 467-477. DOI: 10.1016/j.engfailanal.2015.11.001.

38. Depover T., Wallaert E., Verbeken K. On the synergy of diffusible hydrogen content and hydrogen diffusivity in the mechanical degradation of laboratory cast Fe-C alloys. Materials Science and Engineering: A, 2016, vol. 664, pp. 195-205. DOI: 10.1016/j.msea.2016.03.107. 
39. Lunarska E., Flis J. Effect of hydrogen charging-discharging on the stress-strain relationship for nickel. Scripta Metallurgica, 1984, vol. 18, no. 9, pp. 889-892. DOI: 10.1016/00369748(84)90255-2. 
Подана в журнал: 18.05 .2018

УДК 539.43:669.018

DOI: $10.17804 / 2410-9908.2018 .3 .043-057$

\title{
ВЛИЯНИЕ ПОГРАНИЧНОГО СЛОЯ НА РАСПРЕДЕЛЕНИЕ КОНЦЕНТРАЦИЙ ВОДОРОДА ПРИ ИСПЫТАНИЯХ СТАЛЕЙ НА СТОЙКОСТЬ К ВОДОРОДНОМУ РАСТРЕСКИВАНИЮ
}

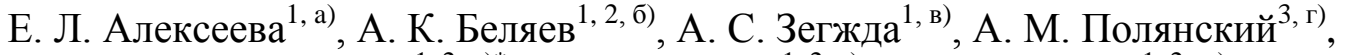 \\ В. А. Полянский ${ }^{1,2, \text { д)* }}$, К. П. Фролова ${ }^{1,2, \text { e) }}$ Ю. А. Яковлев ${ }^{1,2, \text { ж) }}$ \\ ${ }^{1}$ Санкт-Петербургский Политехнический университет Петра Великого, \\ Политехническая ул., 29, Санкт-Петербург, 195294, Российская Федераџия \\ ${ }^{2}$ Институт Проблем Машиноведения РАН, \\ о. Васильевский, пр. Большой, 61, Санкт-Петербург, 199178, Российская Федерация \\ ${ }^{3}$ ООО НПК «Электронные и пучковые технологии», \\ Броневая ул., 6, Санкт-Петербург, 198188, Российская Федерация \\ a) (1D) \\ https://orcid.org/0000-0002-8996-1507, ه alexeeva_ekaterina@mail.ru ; \\ б) (iD https://orcid.org/0000-0002-5934-8138, vice.ipme@gmail.com; \\ в) iD https://orcid.org/0000-0001-8999-5216, ه alexserz@mail.ru ; \\ г) (iD https://orcid.org/0000-0002-6470-9583, ه ampol@electronbeamtech.com; \\ д) (iD https://orcid.org/0000-0002-1199-1028; $ه$ vapol@mail.ru; \\ e) iD https://orcid.org/0000-0003-0376-4463, spfrolova@gmail.com; \\ ж) iD http://orcid.org/0000-0002-5041-0441, \yura.yakovlev@ gmail.com
}

\author{
*Ответственный автор. Электронная почта: vapol@ mail.ru \\ Адрес для переписки: о. Васильевский, пр. Большой, 61, Санкт-Петербург, 199178, Российская Федерация; \\ Тел.: +7-921-748-06-37; факс: +7-812-321-47-71
}

Насыщение водородом является признанным и стандартизированным методом тестирования склонности к водородному растрескиванию и коррозионной стойкости металлов. Насыщение водородом в нейтральном растворе солей, моделирующем морскую воду и подземные воды при барботировании раствора сероводородом, а также в электролите катодным током ионов водорода, является основной промышленной методикой испытаний. Эти испытания являются обязательными для сталей, используемых, например, в газо- и нефтепроводах. В статье описаны результаты исследования распределения концентраций водорода после такого тестирования.

Методом прямых измерений обнаружена сильная неоднородность концентраций водорода, которая ставит под вопрос адекватность методов промышленного тестирования реальным условиям эксплуатации деталей и узлов конструкций в агрессивной среде.

Ключевые слова: насыщение водородом, водородное растрескивание, испытания сталей.

\section{1. Введение}

Влияние водорода на свойства металлов изучают более 150 лет. Первые исследования были посвящены окклюзии водорода металлами [1]. Была разработана аппаратура для измерения концентрации водорода в металле. Затем удалось связать повышенные значения концентрации водорода с уменьшением пластичности металла [2]. С самого начала для исследований применялось искусственное насыщение водородом. В первых работах основными источниками водорода для искусственного насыщения металла были химические реакции металла с кислотой [2] и агрессивная среда [3]. Применялись также методы плавления и нагревания металла в среде газообразного водорода [1].

Boundary layer influence on the distribution of hydrogen concentrations during hydrogen-induced cracking test of steels / E. L. Alekseeva, A. K. Belyaev, A. S. Zegzhda, A. M. Polyanskiy, V. A. Polyanskiy, K. P. Frolova, Yu. A. Yakovlev // Diagnostics, Resource and Mechanics of materials and structures. - 2018. - Iss. 3. - P. 43-57. - DOI: 10.17804/24109908.2018.3.043-057. 
Необходимо отметить, что этот подход на долгие годы приостановил исследования влияния реального, растворенного в металле водорода на свойства отливок и проката, а также внедрение в практику металлургов измерений концентрации водорода. Долгое время разрабатывались технологии улучшения сталей за счет обработки расплавленного металла водородом, и только через 60 лет после работы [2] в 1935 г. появились публикации, связывающие растрескивание отливок, образование флокенов в прокате и снижение пластичности с повышенной концентрацией водорода в стальных слитках [4].

Постепенно разрабатывались стандарты на методы испытаний при исследованиях влияния водорода на разрушение конструкционных материалов. В настоящий момент имеется четыре стандартных способа.

- Насыщение в газообразном водороде $[5,6]$.

- Насыщение в растворе кислоты за счет коррозии или стресс-коррозии [5].

- Катодное наводороживание [5, 7].

- Насыщение водородом в электролите, моделирующем морскую или грунтовую воду или среду транспортируемого природного газа [8].

Все методы стандартизированы, но из-за высокой степени опасности экспериментов по насыщению в газообразном водороде, чаще всего применяется катодное наводороживание.

Необходимо отметить, что метод с таким названием, стандартизованный в [7], позволяет насыщать только плоские образцы, которые можно зажать между уплотнениями двух соседних сосудов с электролитом. Этот стандартизованный метод предполагает контроль полноты насыщения образцов водородом по графику временной зависимости электрического тока между электродами, каждый из которых размещен в своем объеме электролита. Образец играет роль протонно-обменной мембраны между двумя физически разделенными объемами электролита.

Для образцов, имеющих форму цилиндра или прямоугольного параллелепипеда, чаще всего, применяется модификация метода. В качестве одного из электродов выступает сам образец, который нужно быстро насытить водородом. Степень насыщения и равномерность насыщения затем, как правило, не проверяют. Считается, что количество поглощенного водорода пропорционально прошедшему через электролит заряду, а средняя концентрация водорода при одинаковом времени зарядки пропорциональна плотности катодного [9].

Насыщение металла водородом является основным методом исследования воздействия водорода на механические свойства металла. Ежегодно публикуются десятки работ, посвященных исследованиям с использованием этого метода. Поэтому вопрос о распределении концентрации водорода внутри металла в результате насыщения водородом является чрезвычайно важным.

Работ, посвященных этому вопросу, немного [10-14]. Основным методом, применяемым при исследовании распределения концентрации водорода, является математическое моделирование $[10,11,13]$. В работах $[12,14]$ описаны результаты прямых измерений концентрации водорода в медных и стальных образцах, полученных с использованием только одного из четырех методов насыщения - катодного наводороживания.

Прямые эксперименты показывают, что модифицированное катодное наводороживание в случае, когда образец является одним из двух электродов, не позволеют получить равномерного распределения концентраций за разумное время. Для получения однородного распределения концентраций водорода в процессе насыщения необходимо насыщать стальной образец цилиндрической формы диаметром 7 мм в течение 504 ч. Это время почти в 200 раз превышает среднее время наводороживания, которое в большинстве известных экспериментов составляет 2-4 ч. 
В работе [15] установлено, что локальное превышение концентраций водорода всего в 2 раза приводит к разрушению материала. По этой причине объективная картина распределения концентраций водорода после насыщения играет определяющую роль в исследованиях водородной хрупкости материала. Мы провели такое исследование для второго по частоте применения метода наводороживания в электролите. Этот метод описан в стандарте [8] и помимо научных исследований широко применяется при промышленном тестировании сталей на устойчивость к водородной хрупкости.

\section{2. Описание экспериментов, оборудование и приборы}

Для насыщения образцов водородом мы использовали стандартную установку для тестирования на водородное растрескивание, полностью соответствующую [8].

Образцы выдерживались в течение 42-120 ч в деаэрированном растворе на основе дистиллированной воды 5 \% по массе $\mathrm{NaCl} ; 0,5 \%$ по массе $\mathrm{CH}_{3} \mathrm{COOH}$. В процессе проведения теста методом барботирования через раствор продували газообразный сероводород. В рабочей камере создавали и поддерживали постояную концентрациюя сероводорода $2500 \mathrm{мг/л.}$

Время выдержки образцов в электролитическом растворе составляет согласно стандарту [8] 96 ч. Поэтому для исследования динамики процесса насыщения водородом мы изменяли времена наводороживания в диапазоне от 0,44 до 1,6 от стандартного значения. Для насыщения водородом использовали образцы двух типов: прямоугольные параллелепипеды с размерами $20 \times 15 \times 100 \mathrm{mм}^{3}$, вырезанные из листа стального проката, и цилиндрические стержни диаметром 8 мм и длиной 100 мм. Призматические образцы находились в электролите в течение от 42 до 156 ч, цилиндрические образцы - в течение 96 ч. Образцы были изготовлены из листа коррозионностойкой стали 14ХГНДЦ толщиной 17 мм. Все поверхности призматических образцов обрабатывались на шлифовальном станке согласно [8] со средним отклонение профиля порядка 1 мкм. У цилиндрических образцов аналогичная чистота обработки поверхности достигалась на токарном станке.

После насыщения образца водородом и его выдержки на воздухе в течение примерно от 8 до 48 ч из каждого образца, насыщенного водородом, вырезалось по 4 образца для измерений концентрации водорода. После промывки в дистиллированной воде от раствора образцы сушили на воздухе.

Размер призматических образцов соответствовал требованиям [8]. Для измерения концентрации водорода необходимо было использовать более мелкие образцы. Поэтому исходные образцы разрезали ручной пилой на части с размерами $4 \times 6 \times 15 \mathrm{~mm}^{3}$. Нарезка образцов производилась вручную, для того чтобы исключить перегрев материала образцов. Из каждого исходного было вырезано по четыре образца для измерений концентрации водорода. Для исследования влияния поверхностного слоя для каждой серии из четырех мелких образцов у двух удаляли поверхностный слой толщиной 100 и 300 мкм путем ручного сухого шлифования без воды и эмульсий.

Образцы цилиндрической формы после насыщения в течение стандартного времени 96 ч нарезали на цилиндры длинной 10 мм и диаметром 8; 7,88; 7,78 и 7,58 мм. Обработка производилась на токарном станке. Вращение шпинделя производилось с малыми оборотами, чтобы исключить нагрев образцов более чем на 20-30 ${ }^{\circ} \mathrm{C}$. Боковую поверхность образцов диаметром 8 мм не протачивали.

Призматические образцы после насыщения параллельно использовали для измерения параметров трещин, по методике [8]. Для этого на ленточной пиле делали поперечный разрез образцов. После шлифовки, полировки и травления поперечное сечение исследовали под оптическим микроскопом. 
Для измерения концентрации водорода использовали промышленный анализатор водорода АВ-1. Его принцип действия основан на методе высокотемпературной вакуумэкстракции [16-19]. Измерение концентрации водорода производили при температурах экстракции 400, 600 и $800{ }^{\circ} \mathrm{C}$. Эти точки были выбраны специально после проведения предварительных экспериментов. Выбор температуры экстракции производили по аналогии с нашей работой [17].

Ожидаемые концентрации водорода могли иметь большой разброс - от 0,2 до $20\left[\mathrm{млн}^{-1}\right]$. Поэтому калибровкуанализатора проводили по стандартным образцам, так как согласно нашим данным это самый надежный способ. Калибровочные коэффициеты вычисляли как среднее значение по результатам экстракции водорода из трех стандартных образцов с учетом погрешности аттестованного значения, указанного в паспорте. Были выбраны температуры экстракции 400 и $600{ }^{\circ} \mathrm{C}$, так как именно эти значения температур наиболее часто приводятся в литературе как наиболее приемлемые для экстакции диффузноподвижного водорода. При анализе результатов исследований термо-дессорбционных спектров для различных марок сталей [13, 20-22] можно сделать вывод о том, что температура $400{ }^{\circ} \mathrm{C}$ является предельной для диффузинного водорода, а температура $600{ }^{\circ} \mathrm{C}-$ предельной для водорода, который иногда называют диффузно-подвижным.

Температура $800{ }^{\circ} \mathrm{C}$ была выбрана для полного извлечения всего водорода. На эту тему нет единого мнения. Так, в работе [23] рекомендована температура $1000{ }^{\circ} \mathrm{C}$. В работе [24] использована температура $550{ }^{\circ} \mathrm{C}$. В работах [25-27] температура вакуумной экстракции составила $650-680^{\circ} \mathrm{C}$. В работе [28] выбрана температура hot vacuum extruction $500^{\circ} \mathrm{C}$, после этого образцы дополнительно дегазировали в вакууме при температуре $900{ }^{\circ} \mathrm{C}$, чтобы полностью исключить влияние сильно связанного водорода. В работе [29] отмечено, что традиционная температура для извлечения всего водорода методом вакуумной экстракции составляет $650{ }^{\circ} \mathrm{C}$.

Эксперименты, проведенные с цилиндрическими образцами, позволили получить зависимость концентрации водорода, экстрагированного при различных температурах от толщины поверхностного слоя, удаленного с образцов, перед анализом содержания водорода. Практически для всех образцов доля диффузионного водорода в полной концентрации составляла 50 \%, диффузно-подвижного - $25 \%$ и сильно связанного - $25 \%$.

Цлиндрические образцы отличались друг от другпа толщиной удаленного слоя. Это позволяет вычислить распределение средней конценрации водорода по толщине образца. Диаграмма распределения концентрации водорода в зависимости от расстояния от границ слоя до поверхности представлен на рис. 1.

Проведенные исследования показывают, что стандартное насыщение водородом в течение 96 ч приводит к крайне неравномерному распределению водорода внутри образца. Фактически насыщается только тонкий поверхностный слой, имеющий толщину порядка характерного размера зерна.

Как уже отмечалось, для образцов, имеющих форму прямоугольного параллелепипеда, шлифовка поверхности образца осуществлялась вручную, поэтому построение профиля концентраций затруднено из-за значительной неточности удаления поверхностного слоя образцов.

Диаграмма распределения концентрации водорода спустя 12 ч после насыщения образца, выдержанного в коррозионном растворе в течение 72 ч, приведена на рис. 2. 


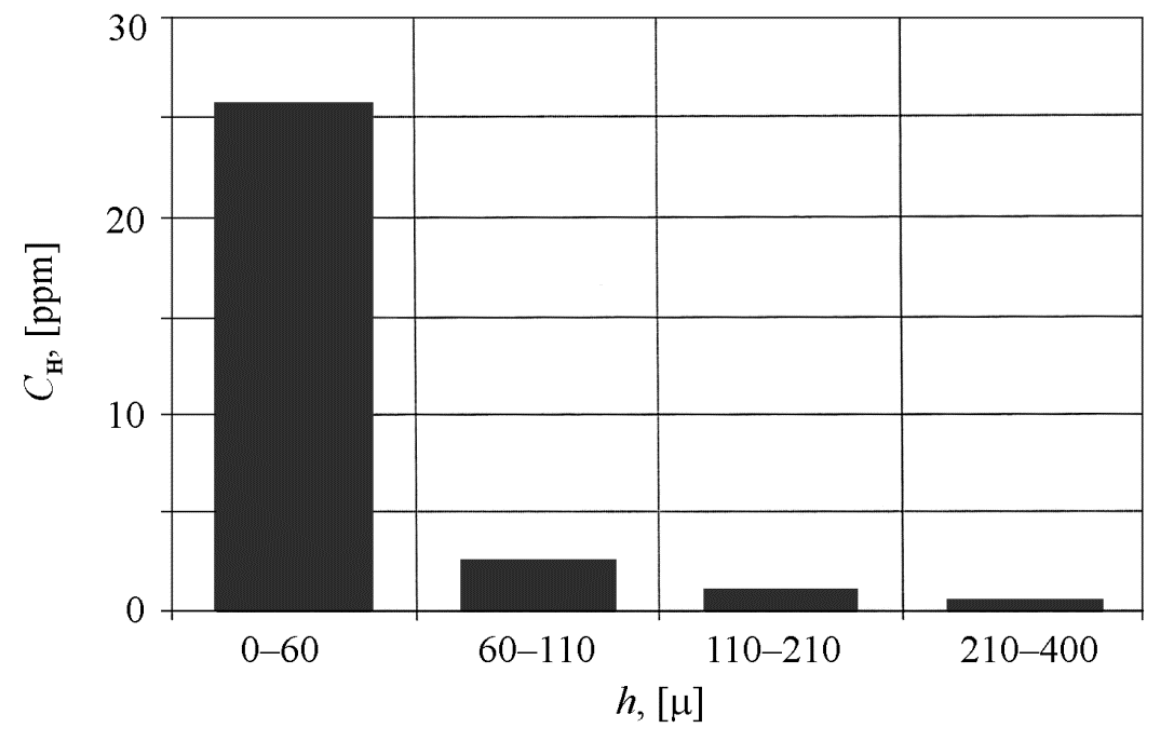

Рис. 1. Расчетные средние значения полной концентрации водорода в поверхностных слоях цилиндрических образцов в зависимости от расстояния $h$ от границ слоя до поверхности образца

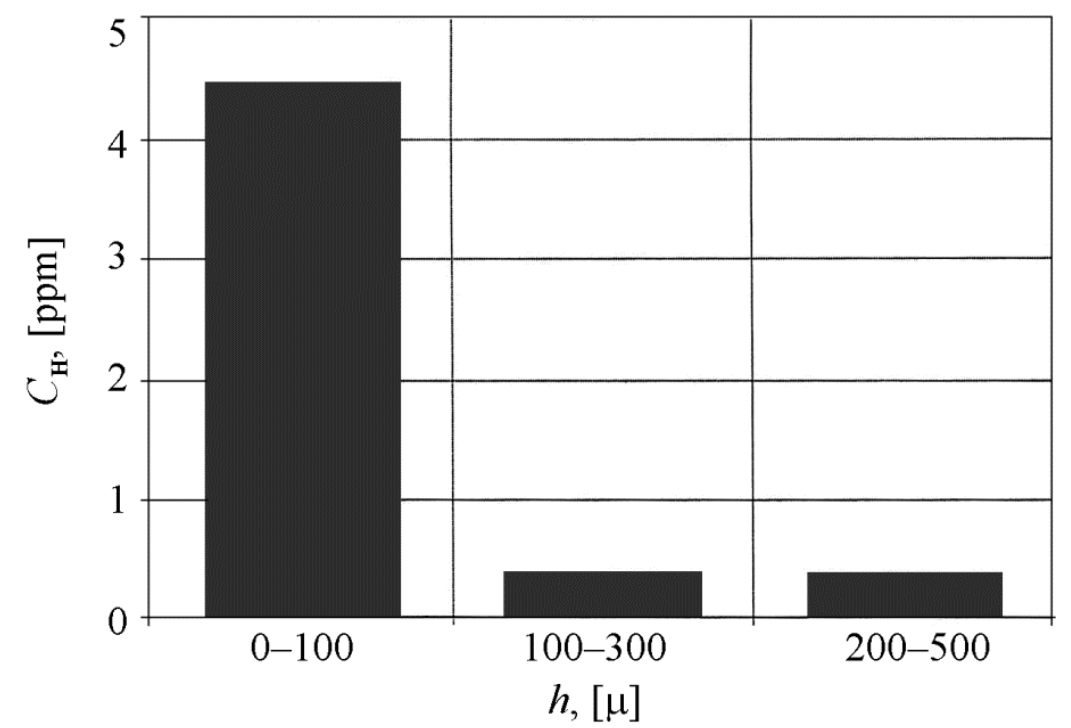

Рис. 2. Расчетные средние значения полной концентрации водорода в поверхностных слоях призматических образцов в зависимости от расстояния $h$ от границ слоя до поверхности образца

Как и для образцов цилиндрической формы, обнаружено многократное превышение содержания водорода в пограничном, поверхностном слое по сравнению с содержанием водорода в объеме образца.

Вместе с тем, водородное растрескивание наблюдалось по всему объему призматических образцов. На рис. 3. представлены фотографии шлифов поперечного разреза образца.

Дополнительно установлено, что водород, накопленный в процессе насыщения, помимо неравномерного распределения концентраций обладает высокой подвижностью по направлению к внешней поверхности образца. Выдержка образца в вакууме в течение 4-х ч привела к 4-6-кратному уменьшению средней концентрации водорода, накопленного при 
насыщении образца. Выдержка образца на воздухе в течение 20 сут приводила к практически полному удалению всего «добавочного водорода», полученного в процессе насыщения образца.

Необходимо заметить, что если время насыщения водородом превышало 72 ч, то суммарная концентрация водорода в пограничном слое стабилизируется. Наблюдался незначительный рост концентрации глубинных слоев образца, который находится в переделах разброса значений «внутренней концентрации» водорода, характерной для исходных, ненасыщенных образцов. То же самое можно сказать и о среднем значении концентрации водорода после длительной выдержки образцов на воздухе.

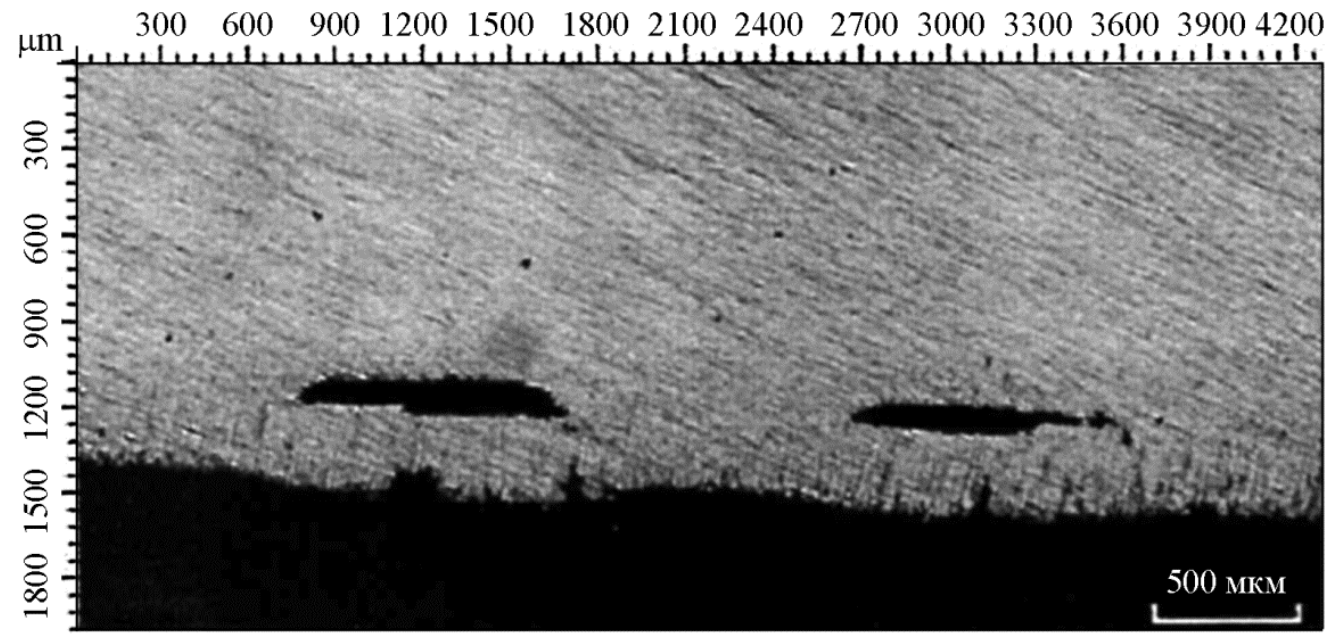

$a$

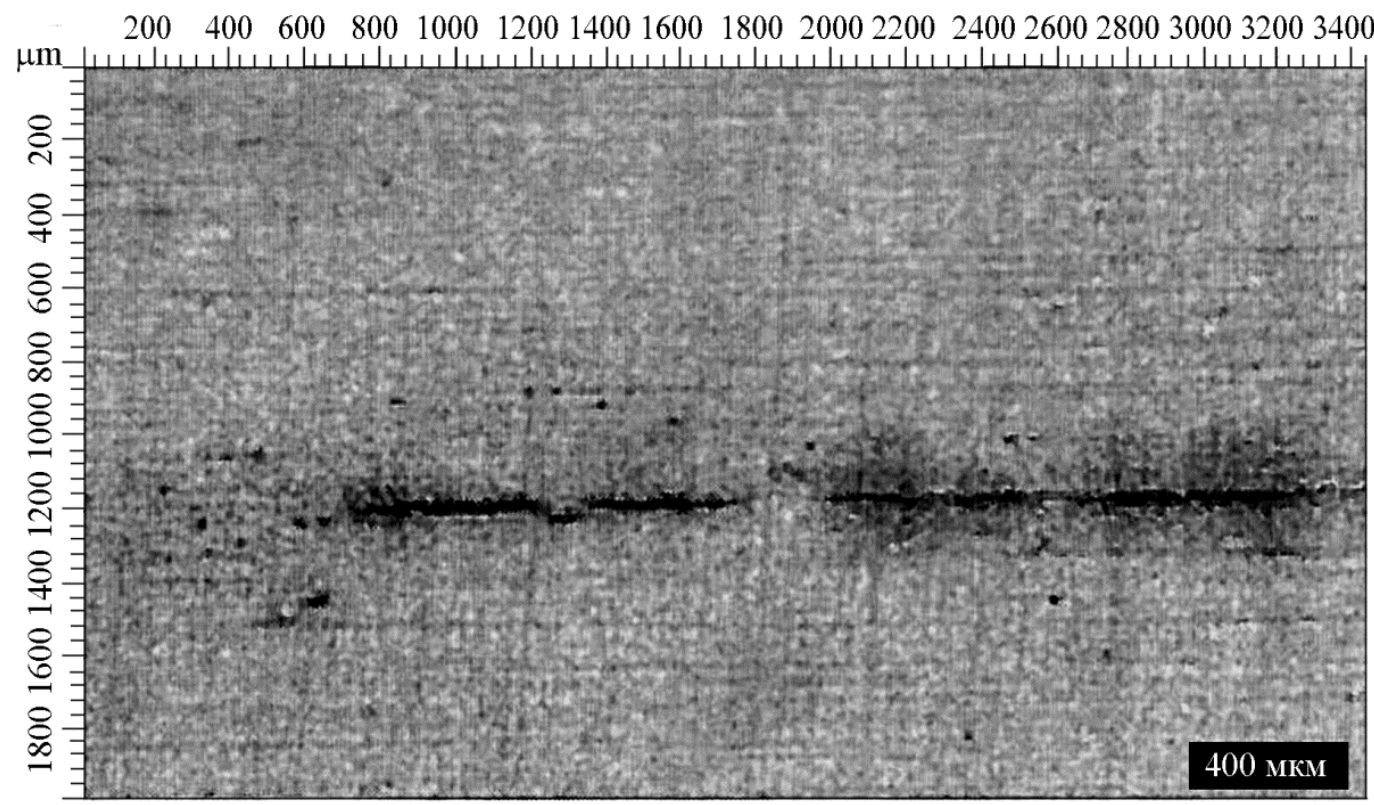

$\sigma$

Рис. 3. Микрофотографии поперечного сечения образцов после насыщения водородом в течение 72 ч 


\section{3. Обсуждение результатов}

Поверхностный эффект, обнаруженный нами в процессе стандартного насыщения, по [8], не описан в известных публикациях. Но эти результаты практически полностью совпадают с результатами, поученными как расчетным, так и экспериментальным путем для cathodic hydrogen charging [10-14]. Там тоже получается очень большая концентрация водорода в поверхностном слое и низкая - внутри. Только после 500 ч насыщения удается получить равномерную концентрацию водорода, но она имеет величину порядка 20 [млн $^{-1}$ ], а в большинстве известных экспериментальных работ средние концентрации водорода оцениваются как единицы $\left[\right.$ млн $\left.^{-1}\right]$.

Разница заключается в том, что нам не удалось зафиксировать тенденцию к росту концентрации водорода во внутренних областях образца при увеличении времени насыщения. Вероятно, это связано с эффектом «экрана», который создает пограничный слой, насыщенный водородом. Влияние этого экрана в случае катодного наводороживания преодолевается за счет приложенной разности потенциалов, хотя и очень медленно. В нашем случае химического наводороживания проникновение водорода блокируется значительно сильнее. При разумных временах насыщения получить более равномерные распределения водорода нам не удалось. Таким образом, мы обнаружили экранирующие свойства пограничного слоя, насыщенного водородом, препятствующие проникновению «дополнительного» водорода вглубь металла.

В нашем случае насыщение водородом длилось до 152 ч, в опытах, описанных в работах $[12,14]$ - до 500 ч. Это на порядок (два) больше тех времен, которые используются большинством исследователей для искусственного насыщения водородом. Обычно время насыщения составляет от 2 до 20 ч. Несмотря на то, что мы и авторы $[12,14]$ проводили опыты с ограниченным кругом материалов, есть основания полагать, что обнаруженный нами эффект носит более общий характер, по крайней мере, это показывают результаты моделирования $[10,11,13]$.

Возникает сразу несколько вопросов, требующих обсуждения.

Первый вопрос, каким образом пограничный слой, толщина которого не превышает $1 \%$ от характерного размера образца, может оказывать такое большое влияние на механические свойства материала. Описаны случаи фактически мгновенного сброса напряжений пластически деформированных образцов, предварительно растянутых в растворе электролита при включении катодного тока [30, 31].

Опыты с образцами, испытавшими пластическую деформацию, показывают, что удаление тонкого поверхностного слоя, толщина которого имеет величину порядка 10-50 мкм, эквивалентно отжигу образца [32]. Развитие трещин при пластическом течении происходит, как правило, в поверхностном слое. Все эти факты говорят о том, что повреждение или ослабление поверхностного слоя играют ведущую роль в развитии трещин, в снижении предела текучести и предела прочности металлов.

Наличие одновременно большого влияния поверхностного слоя на механические свойства металлических образцов при пластической деформации и эффекта быстрого и сильного насыщения пограничного слоя водородом позволяет объяснить сильное и быстрое влияние столь малых средних значений концентраций водорода в образце на механические свойства металлов. Например, буквально через несколько секунд после включения катодного тока в проволоке происходит сброс нагрузки [30, 31], но только в случае, если напряжения находятся в области пластических деформаций.

Вторая проблема заключается в том, что в наших опытах изменение структуры металла, образование трещин и микротрещин наблюдаются по всему сечению образца, а значение средней концентрации водорода внутри образца фактически не отличается от значения концентрации водорода в образце до его насыщения. Эта проблема требует дополнительных ис- 
следований. С одной стороны, диффузия атомарного водорода по междоузлиям, которая считается основным механизмом распространения водорода внутри сталей [33], не должна приводить к полному удалению водорода из монокристаллических зерен, так как это противоречит законам диффузии. Захват и перемещение водорода дислокациями $[34,35]$, который также рассматривается в качестве наиболее вероятного механизма транспорта водорода, также не объясняет наблюдаемых явлений. Дислокации будут захватывать водород из трещин не менее интенсивно, чем из пограничного слоя и распределение концентраций в этом случае должно быть более равномерным.

Получается парадоксальный результат, когда в двух сообщающихся сосудах (в пограничном слое и во внутренних трещинах) водород есть, а в каналах транспорта водорода между ними (поверхности зерен, дислокации, междоузлия кристаллической решетки, вакансии кристаллической структуры и другие общеизвестные ловушки водорода) - нет.

Таким образом, измеренное распределение концентрации водорода противоречит структурным изменениям, наблюдаемым в опытах. Вместе с тем результат не является абсолютно новым, в работе [36] описана аналогичная ситуация. Если насыщать водородом исходные образцы и образцы с предварительным нагружением, то прочность образцов с предварительным нагружением падает быстрее, чем у исходных, а концентрация водорода в предварительно нагруженных образцах после насыщения оказывается меньше, чем в исходных.

Важным фактором является убыль концентрации водорода в образце со временем после его зарядки водородом. По нашим данным скорость убывания «избыточной» концентрации, возникшей в результате насыщения, сильно зависит от внешнего давления. В вакууме скорость уменьшения концентрации водорода была на порядок выше, чем на воздухе.

Концентрация водорода в атмосфере практически равна нулю. Существенная разница в скорости дегазации образца в вакууме и в атмосфере может говорить о том, что водород в молекулярной форме заполняет объем трещин, поэтому уменьшение внешнего давления приводит к дегазации по механизму откачки газообразного водорода из объема, а не по механизму диффузии.

Здесь возникает еще одна проблема, связанная с механизмом накопления молекулярного водорода внутри трещин. Образование трещин требует вложения большой удельной энергии. В случае, когда водород диффундирует в атомарном состоянии, эту энергию можно взять за счет адсорбции атомов водорода на стенках зародышевой трещины. Но в этом случае мы должны фиксировать повышенное значение концентрации водорода внутри образца, так как диффузия - процесс медленный и требующий поступления извне достаточно большой энергии активации диффузии. Однако повышения концентрации внутри образца не наблюдается, что требует дополнительного исследования, тем более, что аналогичные результаты были уже получены ранее. В работах $[36,37]$ отмечено, что нет определенной связи между концентрацией водорода после насыщения и механическими свойствами. В работе [38] отмечено, что недельная выдержка стальных образцов на воздухе после электролитического насыщения водородом приводит к восстановлению пластичности металла.

В работе [39] отмечено, что неравномерность насыщения водородом является большой проблемой при проведении исследований.

Искусственное насыщение водородом широко используют при исследованиях. Водородная хрупкость и другие эффекты, связанные с водородом, часто встречаются в практике. Непонятно, как связано влияние обнаруженного нами эффекта с реальным накоплением водорода и развитием водородной хрупкости, например, при транспортировке газа по газопроводу. Для этого требуется исследовать реальные образцы из газопровода. 
Здесь мы будем ссылаться только на наши собственные исследования. Сравнение образцов стали, вырезанных из стенок газовой трубы, производилось после выдержки их на воздухе от 1 до 6 месяцев. При остаточной концентрации водорода 10-15 ppm мы не обнаружили заметной дегазации образцов в течение этого срока.

Таким образом, реально наблюдаемое медленное накопление водорода может происходить совершенно по другому механизму, чем механизм, использованный при моделировании. Обнаруженный нами эффект существенным образом влияет на соответствие механизмов влияния водорода в случаях модельного и реального накопления внутри металлов. Это позволяет поставить под вопрос многие методы испытаний, в которых поверхностные слои металла удаляются, например катодное наводороживание, по стандарту [7].

\section{4. Заключение}

Экспериментально обнаружен эффект образования особого тонкого пограничного слоя у поверхности образцов при насыщении их водородом в растворе электролита. Концентрация водорода в этом слое в несколько раз превосходит среднюю концентрацию в объеме образцов. Этот результат совпадает с аналогичными результатами, полученными в процессе катодного наводороживания [12, 14].

Помимо большой неоднородности распределения концентрации водорода по глубине образца зафиксирована быстрая дегазация водорода из образцов при их выдержке на воздухе и в вакууме.

Полученные результаты показывают, что делать выводы по результатам механических испытаний образцов, подвергнутых искусственному насыщению водородом, нужно с большой осторожностью, контролируя распределение концентраций водорода путем прямых измерений. Применение расчетного способа может приводить к очень большим ошибкам, так как водород в поверхностном слое создает экран. Этот экран в случае насыщения водородом в растворе электролита препятствует дальнейшему поступлению водорода и его диффузии внутрь образца.

Большинство результатов испытаний, полученных при насыщении водородом в течение времени менее чем 100 ч, можно отнести к чисто поверхностным эффектам.

\section{Благодарность}

Исследование выполнено при поддержке РФФИ, проекты 17-08-00783, 18-08-00201 u 18-31-00329.

\section{Литература}

1. Hadfield R. The occlusion of gases by metals. A general discussion. Introductory address // Transactions of the Faraday Society. - 1919. - Vol. 14. - P. 173-191.

2. Johnson W. H. On some remarkable changes produced in iron and steel by the action of hydrogen and acids // Proceedings of the Royal Society of London. - 1874. - Vol. 23, no. 156-163. P. 168-179.

3. Andrew J. H. The embrittling of iron by caustic soda // Transactions of the Faraday Society. 1914. - Vol. 9. - P. 316-329.

4. Keiichi O. On the importancy of hydrogen-brittleness as a defect in steel qualities // Tetsu-toHagane. - 1938. - Vol. 24, no. 11. - P. 1005-1013. - DOI: 10.2355/tetsutohagane1915.24.11_1005.

5. BS ISO 16573. Steel. Measurement method for the evaluation of hydrogen embrittlement resistance of high strength steels. -2015. 
6. ISO 11114-4. Transportable gas cylinders compatibility of cylinder and valve materials with gas contents. Test methods for selecting metallic materials resistant to hydrogen embrittlement 2005. - P. 4.

7. ISO 17081. Method of measurement of hydrogen permeation and determination of hydrogen uptake and transport in metals by an electrochemical technique. - 2014.

8. ANSI/NACE TM 0284. Evaluation of Pipeline and Pressure Vessel Steel for Resistance to hydrogen-Induced Crack. - 2016. - No. 21215.

9. Akiyama E., Li S. Electrochemical hydrogen permeation tests under galvanostatic hydrogen charging conditions conventionally used for hydrogen embrittlement study // Corrosion Reviews. 2016. - Vol. 34, iss. 1-2. - P. 103-112. - DOI: 10.1515/corrrev-2015-0049.

10. Effect of surface hydrogen concentration on hydrogen embrittlement properties of stainless steels and Ni based alloys / T. Omura, J. Nakamura, H. Hirata, K. Jotoku, M. Ueyama, T. Osuki, M. Terunuma // ISIJ International - 2016. - Vol. 56, no. 3. - P. 405-412. DOI: $10.2355 /$ isijinternational.ISIJINT-2015-268.

11. Hadam U., Zakroczymski T. Absorption of hydrogen in tensile strained iron and high-carbon steel studied by electrochemical permeation and desorption techniques // International Journal of Hydrogen Energy. - 2009. - Vol. 34, no. 5. - P. 2449-2459. - DOI: 10.1016/j.ijhydene.2008.12.088.

12. Martinsson A., Sandstrцm R. Hydrogen depth prole in phosphorus-doped, oxygen free copper after cathodic charging // Journal of Materials Science. - 2012. - Vol. 47, no. 19. P. 6768-6776. - DOI: 10.1007/s10853-012-6592-y.

13. Hydrogen solubility and diffusion in austenitic stainless steels studied with thermal desorption spectroscopy / Y. Yagodzinskyy, O. Todoshchenko, S. Papula, Н. Hдnninen // Steel research international. - 2011. - Vol. 82, no. 1. - P. 20-25. - DOI: 10.1002/srin.201000227.

14. Charging, degassing and distribution of hydrogen in cast iron / R. Wu, J. Ahlstrцm, H. Magnusson, K. Frisk, A. Martinsson // Swerea KIMAB. - 2015. - Vol. 1, no. 14. - P. 1-41.

15. New Metrological Support for Measurements of the Concentration of Hydrogen in Solid Samples / L. A. Konopel'ko, A. M. Polyanskii, V. A. Polyanskii, Yu. A. Yakovlev // Measurement Techniques. - 2018. - Vol. 60, no. 12. - P. 1222-1227. - DOI: 10.1007/s11018-018-1343-3.

16. Konar J., Banerjee N. G. Vacuum heating hydrogen determination in aluminium and aluminium alloys // NML Technical Journal. - 1974. - Vol. 16, no. 1-2. - P. 18-19.

17. Multichannel diffusion vs TDS model on example of energy spectra of bound hydrogen in 34CrNiMo6 steel after a typical heat treatment / A. K. Belyaev, A. M. Polyanskiy, V. A. Polyanskiy, Ch. Sommitsch, Yu. A. Yakovlev. // International Journal of Hydrogen Energy. 2016. - Vol. 41, no. 20. - P. 8627-8634. - DOI: 10.1016/j.ijhydene.2016.03.198.

18. Polyanskiy A. M., Polyanskiy V. A., Yakovlev Yu. A. Experimental determination of parameters of multichannel hydrogen diffusion in solid probe // International Journal of Hydrogen Energy. - 2014. - Vol. 39, no. 30. - P. 17381-17390. - DOI: 10.1016/j.ijhydene.2014.07.080.

19. Application of multichannel diusion model to analysis of hydrogen measurements in solid / D. Yu. Andronov, D. G. Arseniev, A. M. Polyanskiy, V. A. Polyanskiy, Yu. A. Yakovlev // International Journal of Hydrogen Energy. - 2017. - Vol. 42, no. 1. - P. 699-710. DOI: 10.1016/j.ijhydene.2016.10.126.

20. Effect of deformation on hydrogen trapping and effusion in trip-assisted steel / J. H. Ryu, Y. S. Chun, C. S. Lee, H. K. D. H. Bhadeshia, D. W. Suh // Acta Materialia. - 2012. - Vol. 60, no. 10. - P. 4085-4092. - DOI: 10.1016/j.actamat.2012.04.010.

21. Choo W. Y., Lee J. Y. Thermal analysis of trapped hydrogen in pure iron // Metallurgical Transactions A. - 1982. - Vol. 13, no. 1. - P. 135-140. - DOI: 10.1007/BF02642424

22. Ramunni V. P., De Paiva Coelho T., De Miranda P. E. V. Interaction of hydrogen with the microstructure of low-carbon steel // Materials Science and Engineering: A. - 2006. - Vol. 435. P. 504-514. - DOI: 10.1016/j.msea.2006.07.089. 
23. Kim C. D., Loginow A. W. Techniques for investigating hydrogen-induced cracking of steels with high yield strength // Corrosion. - 1968. - Vol. 24, no. 10. - P. 313-318. DOI: $10.5006 / 0010-9312-24.10 .313$.

24. Newman J. F., Shreir L. L. The effect of temperature upon the solubility and diffusion coefficient of cathodic H2 in steel // Corrosion Science. - 1971. - Vol. 11, no. 1. - P. 25-33. DOI: $10.1016 / \mathrm{S} 0010-938 X(71) 80071-9$.

25. Farrell K. Cathodic hydrogen absorption and severe embrittlement in a high strength steel // Corrosion. - 1970. - Vol. 26, no. 3. - P. 105-110. - DOI: 10.5006/0010-9312-26.3.105.

26. Chan S. L. I. Hydrogen trapping ability of steels with different microstructures // Journal of the Chinese Institute of Engineers. - 1999. - Vol. 22, no. 1. - P. 43-53. DOI: $10.1080 / 02533839.1999 .9670440$.

27. Chan S. L. I., Charles J. A. Effect of carbon content on hydrogen occlusivity and embrittlement of ferrite-pearlite steels // Materials Science and Technology. - 1986. - Vol. 2, no. 9. - P. 956-962. - DOI: 10.1179/mst.1986.2.9.956.

28. Hydrogen solubility in pure iron and effects of alloying elements on the solubility in the temperature range 20 to $500 \mathrm{C} / \mathrm{W}$. Y. Choo, J. Y. Lee, C. G. Cho, S. H. Hwang // Journal of Materials Science. - 1981. - Vol. 16, no. 5. - P. 1285-1292. - DOI: 10.1007/BF01033843.

29. Jenkins N., Hart P., Parker D. H. An evaluation of rapid methods for diffusible weld hydrogen // Welding Journal-Including Welding Research Supplement. - 1997. - Vol. 76, no. 1. - P. 1-6.

30. Lunarska E., Flis J. Effect of hydrogen charging-discharging on the stress-strain relationship for nickel // Scripta Metallurgica. - 1984. - Vol. 18, no. 9. - P. 889-892. - DOI: 10.1016/00369748(84)90255-2.

31. Lunarska E. Changes in the strain hardening rate of iron and steel during absorption and desorption of hydrogen under cathodic charging and discharging conditions // Scripta Metallurgica. - 1981. - Vol. 15, no. 10. - P. 1077-1082. - DOI: 10.1016/0036-9748(81)90161-7.

32. Pearson W. B. Miscellaneous aspects of lattice-spacing variation : chapter V // A Handbook of Lattice Spacing and Structures of Metals and Alloys : International Series of Monographs on Metal Physics and Physical Metallurgy. - Vol. 4. - Oxford : Pergamon, 1958. - P. 68-75.

33. Hydrogen embrittlement of a single crystal of iron on a nanometre scale at a crack tip by molecular dynamics / Z. Hu, S. Fukuyama, K. Yokogawa, S. Okamoto // Modelling and Simulation in Materials Science and Engineering. - 1999. - Vol. 7, no. 4. - P. 541.

34. Pressouyre G. M. Trap theory of hydrogen embrittlement // Acta Metallurgica. - 1980. Vol. 28, no. 7. - P. 895-911. - DOI: 10.1016/0001-6160(80)90106-6.

35. Song J., Curtin W. A. A nanoscale mechanism of hydrogen embrittlement in metals // Acta Materialia. - 2011. - Vol. 59, no. 4. - P. 1557-1569. - DOI: 10.1016/j.actamat.2010.11.019.

36. . Influence of $\mathrm{H} 2 \mathrm{~S}$ interaction with prestrain on the mechanical properties of high-strength X80 steel / Y. Chen, S. Zheng, J. Zhou, P. Wang, L. Chen, Y. Qi // International Journal of Hydrogen Energy. - 2016. - Vol. 41, no. 24. - P. 10412-10420. - DOI: 10.1016/j.ijhydene.2016.01.144.

37. Measurement of hydrogen and embrittlement of high strength steels / J. Sanchez, S. F. Lee, M. A. Martin-Rengel, J. Fullea, C. Andrade, J. Ruiz-Hervias // Engineering Failure Analysis. 2016. - Vol. 59. - P. 467-477. - DOI: 10.1016/j.engfailanal.2015.11.001.

38. Depover T., Wallaert E., Verbeken K. On the synergy of diffusible hydrogen content and hydrogen diffusivity in the mechanical degradation of laboratory cast Fe-C alloys // Materials Science and Engineering: A. - 2016. - Vol. 664. - P. 195-205. - DOI: 10.1016/j.msea.2016.03.107.

39. Lunarska E., Flis J. Effect of hydrogen charging-discharging on the stress-strain relationship for nickel // Scripta metallurgica. - 1984. - Vol. 18, no. 9. - P. 889-892. - DOI: 10.1016/00369748(84)90255-2. 